\title{
An in vivo strategy for knockdown of circular RNAs
}

\author{
Nagarjuna Reddy Pamudurti (1), Ines Lucia Patop ${ }^{1}$, Aishwarya Krishnamoorthy', Reut Ashwal-Fluss ${ }^{2}$, Osnat Bartok ${ }^{2}$ and \\ Sebastian Kadener ${ }^{1,2}$
}

\begin{abstract}
Exonic circular RNAs (circRNAs) are highly abundant RNAs generated mostly from exons of protein-coding genes. Assaying the functions of circRNAs is not straightforward as common approaches for circRNA depletion tend to also alter the levels of mRNAs generated from the hosting gene. Here we describe a methodology for specific knockdown of circRNAs in vivo with tissue and cell resolution. We also describe an experimental and computational platform for determining the potential off-target effects as well as for verifying the obtained phenotypes. Briefly, we utilize shRNAs targeted to the circRNA-specific back-splice junction to specifically downregulate the circRNA. We utilized this methodology to downregulate five circRNAs that are highly expressed in Drosophila. There were no effects on the levels of their linear counterparts or any RNA with complementarity to the expressed shRNA. Interestingly, downregulation of circCtrip resulted in developmental lethality that was recapitulated with a second shRNA. Moreover, downregulation of individual circRNAs caused specific changes in the fly head transcriptome, suggesting roles for these circRNAs in the fly nervous system. Together, our results provide a methodological approach that enables the comprehensive study of circRNAs at the organismal and cellular levels and generated for the first time flies in which specific circRNAs are downregulated.
\end{abstract}

\section{Introduction}

Exonic circular RNAs (circRNAs) are a highly abundant type of RNA produced through circularization of specific exons in a process known as back-splicing ${ }^{1-4}$. circRNAs are expressed in tissue- and development stage-specific ways, independently of the expression of the hosting gene $^{5}$. Indeed, abundant circRNAs can control expression of their hosting genes in cis by deviating transcription toward circRNA production ${ }^{6}$. circRNAs are produced by the spliceosome and their production is driven by inverted repeat sequences in the RNA or by RNA-binding proteins ${ }^{6-12}$. In addition, some circRNAs also produce proteins $^{13-15}$. The circRNA-encoded peptides usually share start codons with their hosting genes and might be important in synapse and muscle functions ${ }^{13}$.

Correspondence: Sebastian Kadener (skadener@brandeis.edu)

${ }^{1}$ Biology Department, Brandeis University, Waltham, MA 02454, USA

${ }^{2}$ Biological Chemistry Department, Silberman Institute of Life Sciences, The

Hebrew University of Jerusalem, 91904 Jerusalem, Israel

These authors contributed equally: Nagarjuna Reddy Pamudurti, Ines

Lucia Patop
circRNAs are particularly enriched in neural tissue ${ }^{16-19}$. Moreover, circRNA levels increase with age in the brains of mice and flies as well as in worms ${ }^{18,20,21}$ and are affected by neuronal activity ${ }^{19}$. These observations suggest important roles for circRNAs in the brain. Indeed, mice depleted of the circRNA CDR1as have abnormal gene expression in the brain and specific behavioral defects $^{22}$. Recent work has identified a handful of circRNAs that function in trans: the circRNAs derived from CDR1as and sry likely regulate miRNA function and/or localization $^{22-25}$. Other circRNAs titrate or transport proteins and might be important for cancer development ${ }^{6,26,27}$. circRNAs can also mediate responses to viral infections ${ }^{28-31}$.

Despite steady advances, the circRNA field faces one main obstacle: it is usually not possible to downregulate the amount of a given circRNA without altering the levels of the linear RNA produced from the same hosting gene. Also, as circRNA production can compete with linear RNA splicing, it is difficult to separate the potential cis and trans functions of the circRNA. Recently, the 
Rajewsky lab generated the first animal (mouse) without a single circRNA (CDR1as). They did so by deleting the locus from which circRNA is generated ${ }^{22}$. This was possible because the CDR1as locus is unusual as it does not encode a linear RNA. In cell culture studies, shRNAs (mostly transiently transfected) can alter circRNA levels, but shRNA-mediated knockdown is often inefficient (especially in mammalian systems) and can result in undesired silencing of the linear mRNA transcript encoded by the same locus or in other off-target effects ${ }^{4}$. A recent study utilized lentiviral transduction of a circRNAdirected shRNA to knockdown a specific circRNA in vivo in the mouse orbitofrontal cortex. Interestingly, they found a reduced cognitive flexibility in these mice even when they achieved only a $40 \%$ reduction on the levels of the targeted circRNA ${ }^{32}$.

Another way to modulate the levels of a circRNA is by deleting the intronic sequences responsible for exon circularization $^{33-35}$. This approach can be a great option while working with a single circRNA but it is laborious and would be difficult (or impossible) to use it to globally screen for functions of circRNAs and it does not allow for tissue-specific resolution. In addition, one cannot differentiate between cis and trans functions of circRNAs using this technique, and it can probably only be used to identify functions of circRNAs that act in trans. This is because impairing the biogenesis of circRNAs whose production regulates the expression of the hosting gene will result by definition in changes on the levels of the linear mRNA.

Here we adapted the use of shRNAs in order to specifically target circRNAs with cell and tissue resolution in Drosophila. Our methodology takes advantage of the functional separation of the miRNA and shRNA systems in flies and utilizes shRNAs generated by miRNA-like precursors $^{36}$ and targeted specifically to the back-splicing junction. This allows us to achieve specific knockdown without altering the levels of the mRNA generated from the host gene. We demonstrated the utility of this method by targeting five highly expressed circRNAs. To determine potential off-target effects, we generated and sequenced RNA-seq libraries from the heads of these and control strains. We did not detect significant changes in the expression of any of these potential off-targets (mRNAs with incomplete but perfect or seed-like complementarity to the expressed shRNA). Interestingly, downregulation of one of the targeted circRNAs, circCtrip, resulted in developmental lethality that we recapitulated with a second shRNA targeting the same circRNA. Moreover, we found that downregulation of individual circRNAs led to specific changes in the fly head transcriptome, suggesting specific roles for these particular circRNAs in regulation of gene expression. Together, our results provide a methodological approach that enables the comprehensive study of circRNAs at the organismal and cell levels.

\section{Results}

circRNAS can be specifically downregulated by miRNAderived shRNAs in vivo

To knockdown circRNAs in vivo, we generated flies that express shRNAs directed against individual circRNAspecific back-splicing junctions (Fig. 1a). For shRNA expression, we utilized a vector based on a miRNA-like precursor $\left(\mathrm{miR}-1^{36}\right)$. For these experiments, we chose to target five circRNAs with high levels of expression in fly heads based on previous work ${ }^{6}$. In all cases we expressed the shRNAs using the GAL4/UAS system that allows temporal and spatial control of expression. We generated flies expressing the transgene under the control of a constitutive driver (actin-Gal4). In four of the five cases, we obtained viable flies (Supplementary Fig. S1a). We then evaluated the efficiency of the circRNA knockdown in fly heads and observed a specific and strong reduction in levels of the targeted circRNAs in this body part. For the four viable strains, expression of the shRNA led to more than $75 \%$ reduction in the level of the targeted circRNA (Fig. 1b).

\section{Most circRNA knockdown lines do not display identifiable off-targets effects}

To further determine the specificity of the knockdowns, we measured the expression level of the linear RNAs generated from the genes hosting each circRNA by qRTPCR. Indeed, expression of the shRNA did not change their levels, demonstrating the knockdown is specific for the circular form of the RNA (Fig. 1c). For these lines we also measured the levels of the other circRNAs and confirmed that the knockdown is specific for the targeted circRNA (Fig. 1d).

In addition, and to determine globally off-targets of the expressed shRNAs, we generated and sequenced $3^{\prime}$-end libraries from heads of the four knockdown strains that did not cause developmental lethality. The 3'-RNA-seq technique is highly reliable for determining mRNA levels of low abundance mRNAs ${ }^{37}$. As determined by qRT-PCR (see above) we observed that for all the assayed strains, the shRNAs did not significantly alter the levels of the mRNAs produced from the same locus (Fig. 1c; Supplementary Fig. S1b and Table S2).

Despite the lack of effect of the shRNAs on the level of the linear mRNAs, it is possible that translation of the hosting mRNAs could be impacted. We tested this possibility by analysis of two strains for which there are antibodies available against the proteins encoded by their linear counterpart RNAs. Expression of the shRNAs targeting the back-splice junctions of circPkn and circPlexA strongly downregulated the targeted circRNAs without reducing the levels of PKN or PLEXA proteins, demonstrating that the shRNA does not target the linear RNAs at the translational level (Fig. 1e). PKN levels seems to be 
a.

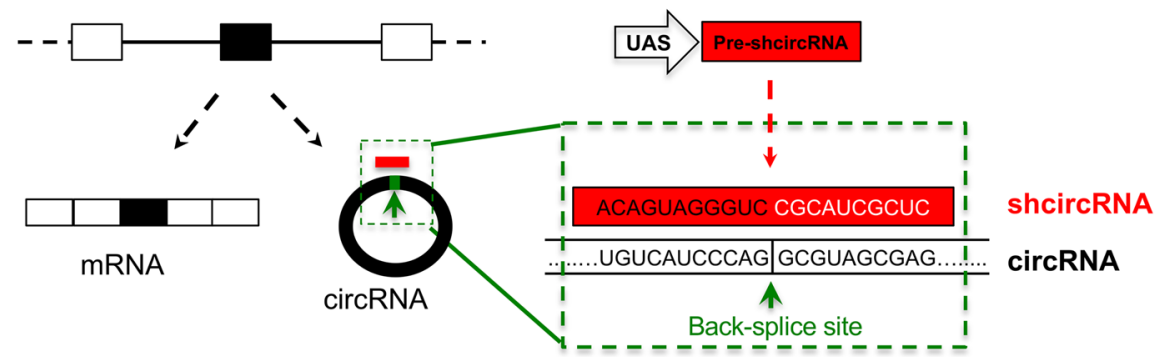

b.

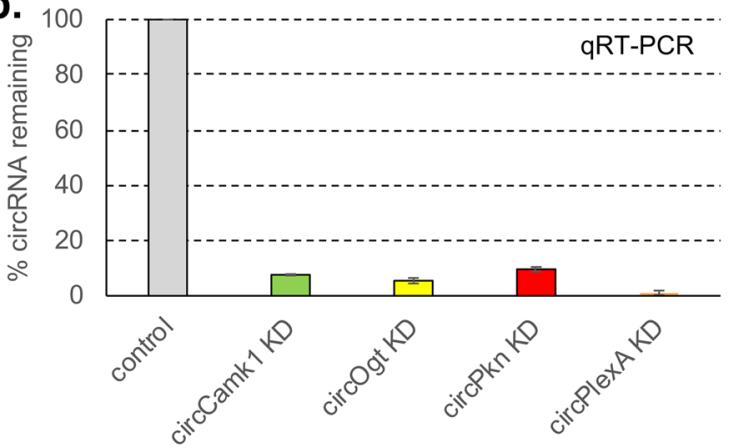

c.

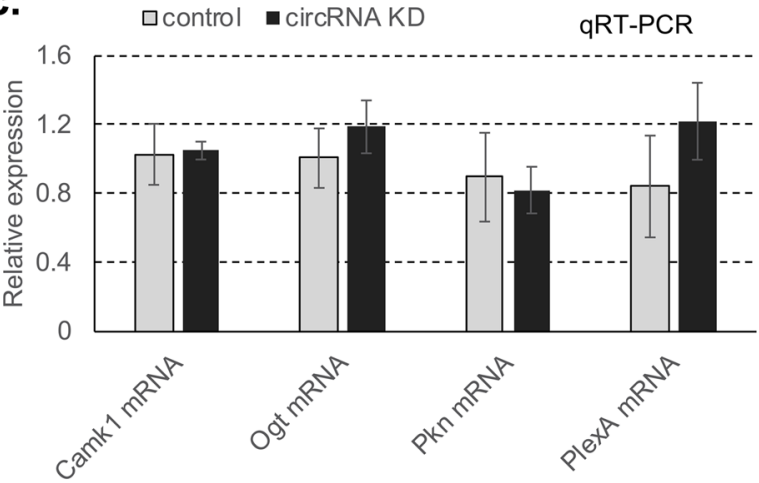

e.
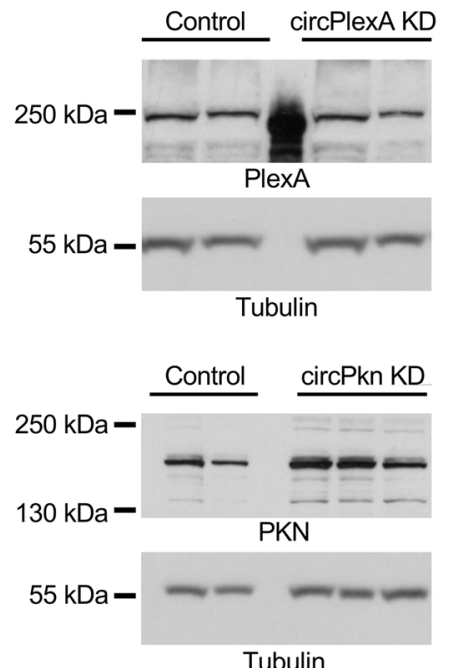

Tubulin

d.

qRT-PCR

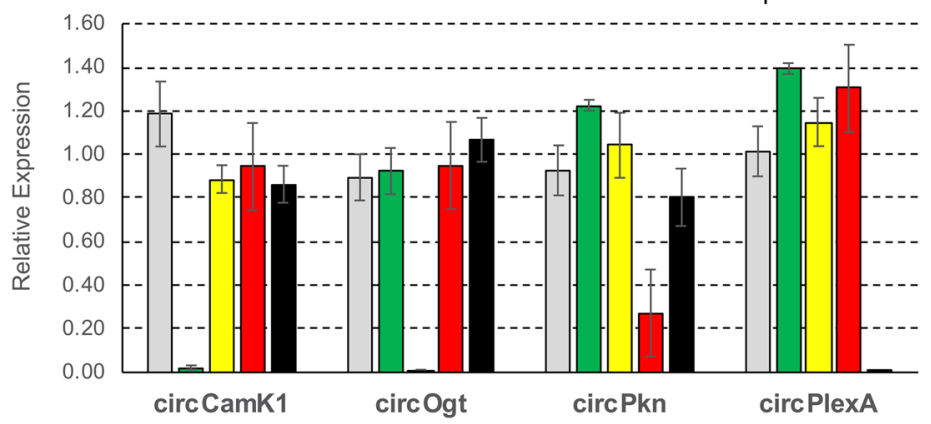

口Control

acircCamK1 KD

口circOgt KD

acircPkn KD

- circPlexA KD

Fig. 1 circRNAs can be specifically downregulated by genetically encoded shRNA in vivo. a Scheme of the shRNA strategy to knockdown circRNAs in vivo. ShRNAs are generated from a UAS-based miR-1 like precursor (see Materials and methods). $\mathbf{b}$ Relative levels of the targeted circRNAs in fly heads expressed as percentage of control (actin-Gal4 flies). Levels of the indicated circRNAs were assess by qRT-PCR using the rp49 mRNA as normalization control ( $n=3$, error bars represent standard error of the mean). c Relative expression levels of the indicated mRNAs in the four knockdown strains. Levels were normalized to mean expression in control line (actin-Gal4 flies). In all cases we utilized fly heads as source of material and assessed the levels of the mRNAs by qRT-PCR using rp49 as normalization control ( $n=3$, error bars represent standard error of the mean). d Levels of the indicated circRNAs in the four knockdown strains. In all cases we utilized fly heads as source of material and assessed the levels of the circRNAs by qRT-PCR using rp49 as normalization control ( $n=3$, error bars represent standard error of the mean). e Levels of the PKN and PLEXA proteins (and Tubulin as loading control) as assayed by western blot in heads of control (actin-Ga/4), circPkn and circPlexA KD strains. 
slightly upregulated. If that is the case, this might be due to a direct or indirect effect of circPkn downregulation rather than to a non-specific effect of the shRNA. In sum, these experiments demonstrate that specifically targeting circRNA junctions with an shRNA depletes the circRNA without any impact on hosting gene expression.

In addition, shRNAs could have non-specific effects on other mRNAs. To evaluate this possibility, we determined whether downregulated mRNAs in shRNA-expressing strains were enriched for sequence complementary to the seed of the shRNA or shRNA* utilizing the SYLAMER algorithm ${ }^{38}$. In none of our lines the downregulated mRNAs were enriched for any relevant seed sequences (Fig. 2a).

It is also possible that shRNAs could target mRNAs with more extensive base complementarity in any part of the mRNA. To test this possibility, we identified mRNAs with 12 or more bases complementary to the shRNAs and determined whether any of them were downregulated upon expression of the shRNA. Indeed, none of the mRNAs with base complementarity to the shRNA display significant changes in expression upon silencing of the circRNA (Fig. 2b; Supplementary Table S3). Together these results demonstrate that these shRNAs act specifically and do not display observable off-target effects.

\section{circRNA knockdown provokes specific changes in the head transcriptome}

We then analyzed more carefully the mRNAs differentially expressed in fly heads upon expression of the four non-lethal shRNAs. We found that downregulation of specific circRNAs provoked specific changes in the head transcriptome (Fig. 3a; Supplementary Tables S2 and S4). For most of the circRNA knockdown strains, genes belonging to specific Gene Ontology (GO) terms were significantly enriched in the differentially expressed genes (Fig. 3b; Supplementary Table S5). For example, knockdown of circCamk1 provoked changes in genes related with sensory perception of smell and sugar metabolism. Interestingly, the Camk1 mRNA is enriched in the eyes ${ }^{39}$ and encodes a protein that is involved in olfactory receptor signaling ${ }^{40}$. Upon knockdown of circOgt, we found that differentially expressed genes were enriched for signaling receptor activity, while upon knockdown of circPkn we found GO terms related to muscle function and response to pheromone along with sensory perception (as observed in the circCamk1 KD strain). The differentially expressed genes in flies in which circPlexA was knocked down were enriched for GO terms related to photoreceptor activity. Remarkably, the PlexA gene is involved in regulation of photoreceptor cell axon guidance $^{41}$. Furthermore, we found some GO terms shared
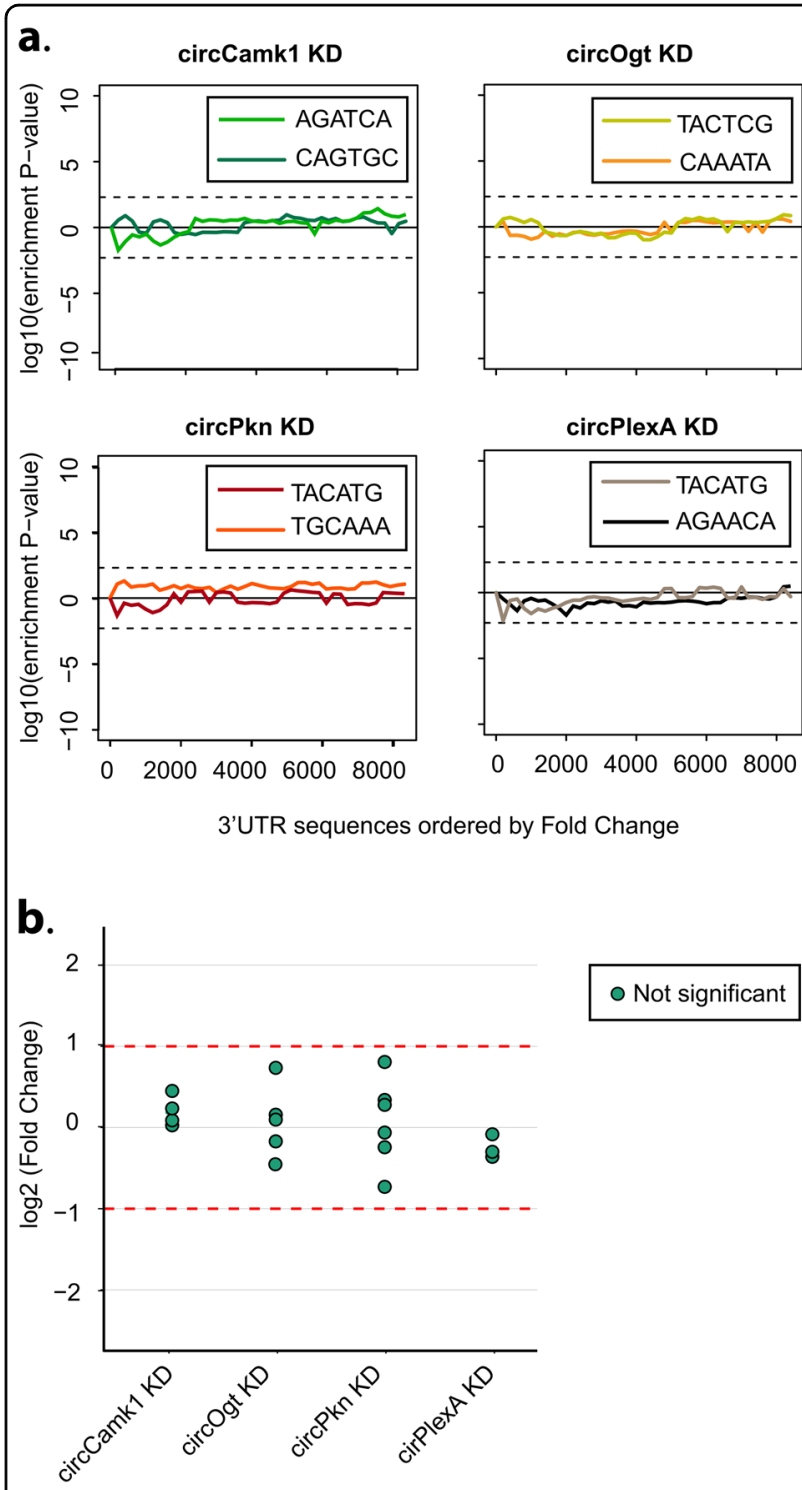

Fig. 2 shRNAs targeting circRNAs have no detectable off-targets. a Assessment of off-targets of the indicated shRNAs by SYLAMER. Seed enrichment was calculated for the genes differentially expressed upon downregulation of circCamk1, circOgt, circPkn, or circPlexA. shRNA and shRNA* seed sequences shown in different colors. Genes were sorted from downregulated to upregulated. For all knockdowns we did not observed any shRNA seed enriched among the downregulated genes indicating absence of detectable off-targets. b Expression of putative off-targets genes in circRNA KD lines. Potential off-targets genes were selected using blast of the shRNA sequence against the Drosophila transcriptome. $3^{\prime}$ RNA-seq data was used to determine the expression level of each putative off-targets gene relative to control line (actin-Gal4, $n=3$ ). Differential genes expression was performed using a generalized linear model with negative binomial distribution (see Materials and methods for details). None of the mRNAs displayed statistically significant differences (log2 (fold change) $>1$ or $<-1$ and corrected $p$ value $<0.05$ ). $X$-axis presents $\log 2$ (fold change). None of the potential off-targets detected in the sequencing is differentially expressed. 
a.
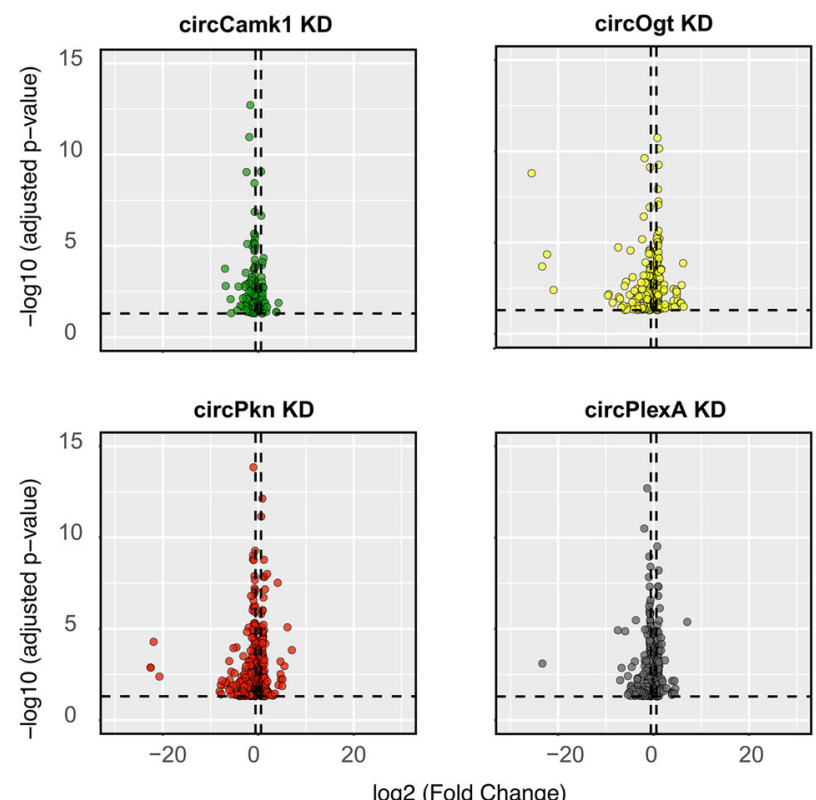

b.
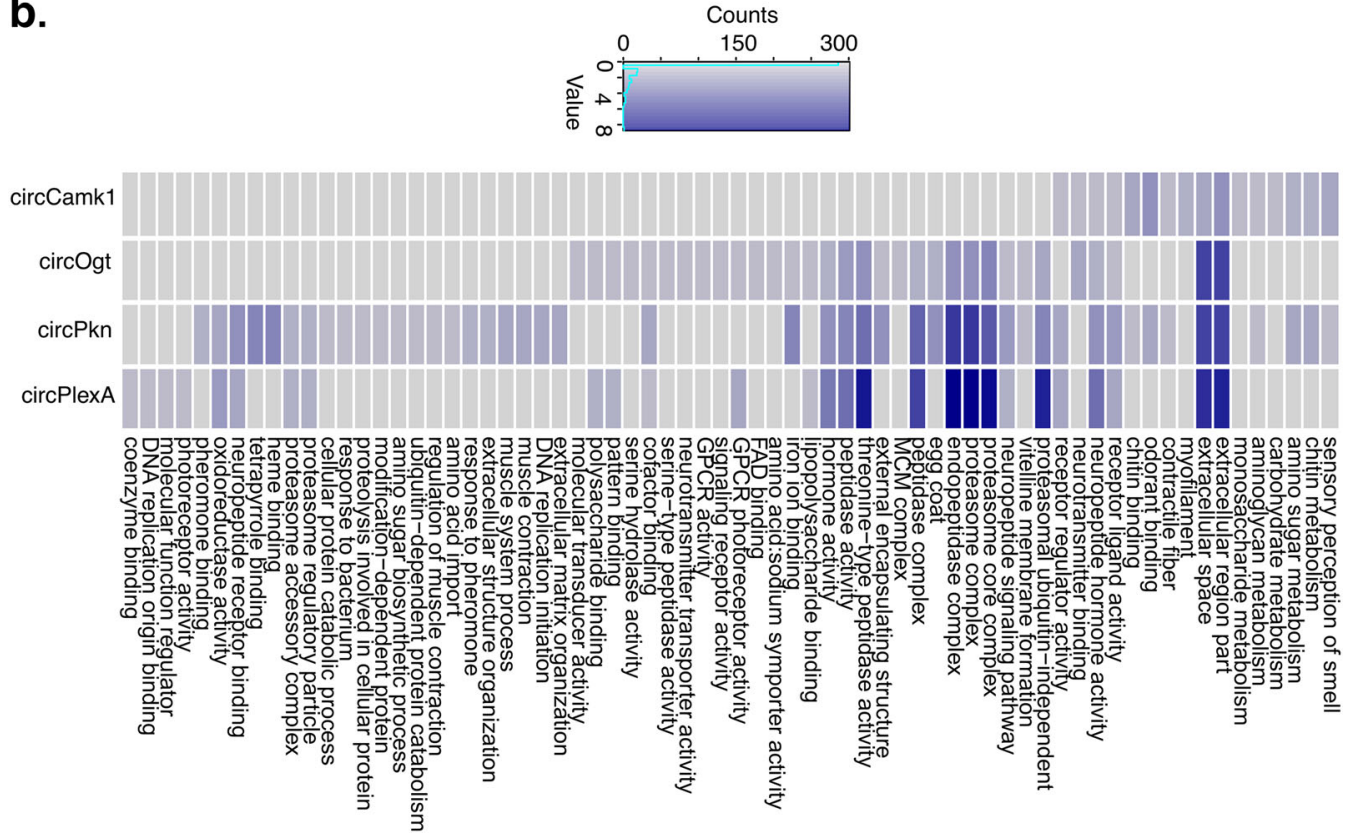

Fig. 3 circRNA KD modulates specific set of gene expression. a Differentially expressed genes in different circRNA KD flies in comparison to control flies (actin-Ga/4). Each plotted point represents the differential expression result for an individual gene. The $x$-axis shows the log 2 of the fold change between the expression measurement in the knockdown and the control strain fly. The $y$-axis shows the $-\log 10$ of the adjusted $p$-value for the same comparison. In dashed lines we represent the limits $y=\log 10(p$ value $=0.05)$ and $x=\log 2$ (fold change $=1.5$ ). Complete results are in Supplementary Table S4. b Gene Ontology (GO) terms significantly enriched (FDR $<0.1$ ) among genes differentially expressed between the heads of control and each circRNA-KD fly strain. Color represent $-\log 10$ of adjusted $p$-value. Redundant terms were curated manually, and names were simplified for clarity reason. Complete results are in Supplementary Table S5.

between the differentially expressed genes upon knockdown of the specific circRNAs. Among them were neurotransmitter binding, receptor regulator activity and some terms related with development and metabolism.
Overall, the gene expression results suggest that these circRNAs might have different physiological functions, some of which might overlap with the function of their linear counterparts. 
Knockdown of circCtrip results in developmental lethality

Expression of an shRNA designed to deplete circCtrip led to very strong developmental lethality (Supplementary Fig. 1a). Moreover, the few flies that did eclose from this strain (actin-Gal4; UAS-shcircCtrip/CG14656) had locomotion defects and died within a few days (Supplementary Movie S1). We utilized a GFP-labeled balancer chromosome to determine the timing of the lethality. We found that expression of the shRNA against circCtrip caused lethality at the pupal stage (Fig. 4a). We then measured the levels of circCtrip and a circRNA that while silenced does not lead to developmental lethality (circCamk1) at different developmental stages (Fig. 4b). While the levels of linear ctrip mRNA have a peak of expression at the embryonic stage, circCtrip is expressed only after embryonic stage and display the higher expression at the early pupal stage. On the other hand, expression of circCamk 1 is more uniform across the surveyed developmental stages. Consistent with this, analysis of previously published gene expression data of flies over the course of development ${ }^{18}$ showed that circCtrip is highly expressed in the central nervous system at the larval and pupal stages (Supplementary Fig. 1c).

To rule out off-target effects, we generated a new fly line expressing a second shRNA targeting circCtrip. The new shRNA was designed to be perfectly complementary to the circRNA junction, but the target site was shifted and hence potential off-target effects should be reduced (Fig. 4c). We found that expression of the second shRNA construct targeting circCtrip also resulted in developmental lethality when expressed under the actin-Gal4 driver (Fig. 4d). To confirm that expression of these two shRNAs did indeed downregulate circCtrip, we generated flies in which the shRNAs were expressed specifically in the fly central nervous system (CNS) by using the elavGal4 driver. Using this driver allowed us to bypass the developmental lethality due to the lower and more restricted knockdown. We observed a strong (around 90\%) downregulation of circCtrip in both shRNAexpressing lines (Fig. 4e).

To completely rule out the possibility that the developmental lethality of the circCtrip shRNAs is due to offtarget effects, we prepared and sequenced $3^{\prime}$-RNA-seq libraries from heads of the CNS-specific circCtrip KD and control strains. We did not detect significant effects of either shRNA on the levels of ctrip mRNA (Fig. 4f; Supplementary Table S6). In addition, the genes with complementarity to these shRNAs were not differentially expressed upon the expression of either shRNA targeting circCtrip (Fig. 4g; Supplementary Tables S7 and S8). Last but not least, we used SYLAMER to look for potential miRNA-like effects of the shRNAs or shRNAs" generated from the circCtrip shRNA constructs. We indeed did not observe any enrichment for mRNAs containing the seed sequence among those downregulated upon expression of any of the two shRNAs against circCtrip (Fig. 4h, i). These results demonstrate that shRNAs targeting circCtrip have no detectable off-target effects in fly heads and that this circRNA is necessary for proper development.

To try to understand the potential functions of circCtrip, we further analyzed the gene expression changes observed upon knockdown of circCtrip in the central nervous system. Interestingly, we found that genes differentially expressed upon circCtrip knockdown were enriched for genes related to metabolism, cuticle and chitin development, and proteasome activity (Supplementary Tables S8 and S9). When manually inspecting the most de-regulated genes in these flies, we found genes encoding for heat-shock protein, cuticular proteins, and metabolism related proteins (Supplementary Table S8). These results suggest that deregulation of these pathways might be related to the lethality observed in circCtrip KD flies, but further experiments are necessary to confirm this.

\section{Discussion}

In this manuscript, we adapted the use of genetically encoded shRNAs to downregulate for the first time circRNAs in Drosophila. To do so, we designed miRNAderived shRNAs that target the circRNA-specific backsplicing junctions of five highly expressed circRNAs. Expression of these shRNAs led to very strong and specific downregulation of the targeted circRNAs with no effects on the levels of the linear RNA and in two cases protein generated from the same loci. In addition, we did not detect any changes in expression of genes with complementarity to the shRNA or with seed-like sequences in their $3^{\prime}$ UTRs, demonstrating that the utilized shRNAs have no detectable off-target effects. We utilized this approach to downregulate five highly expressed circRNAs in vivo. We found that downregulation of circCtrip resulted in developmental lethality that was recapitulated with a second shRNA. Moreover, downregulation of individual circRNAs caused specific changes in gene expression. In sum, the methodology described here will allow investigation of the functions of circRNAs in vivo, and we have established quality standards for detection of specific and off-target effects of shRNAs when targeting circRNAs.

Although circRNAs are very abundant, especially in neural tissue, only a few studies have assessed the functionalities of these molecules in vivo. A main obstacle for performing functional experiments is the lack of tools for specifically manipulating circRNA levels. As expression of circRNAs generally depends on inverted RNA repeats or RNA binding proteins, it is theoretically possible to modulate circRNA levels by deleting those elements from the DNA (and hence RNA) by CRISPR. However, this 
a.

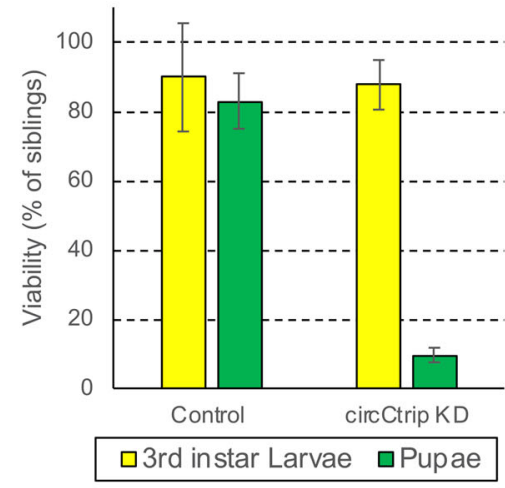

c.

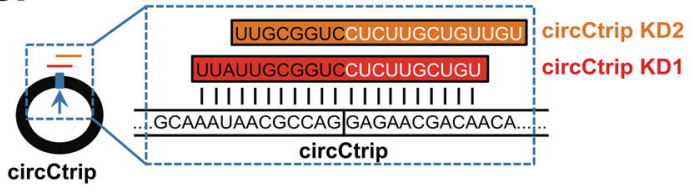

e. circCtrip

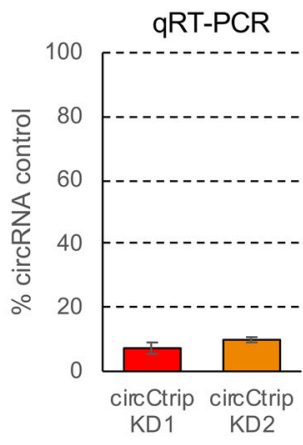

f. ctrip mRNA

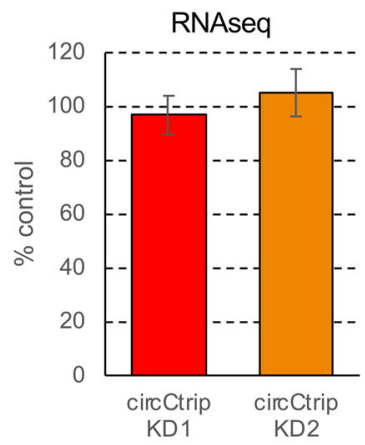

b.

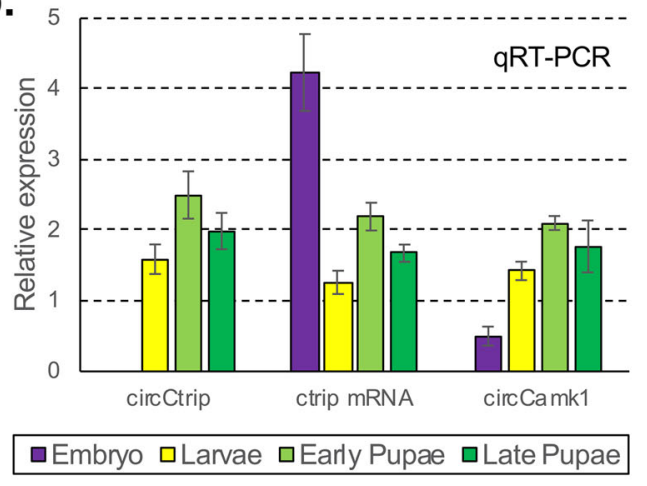

d.

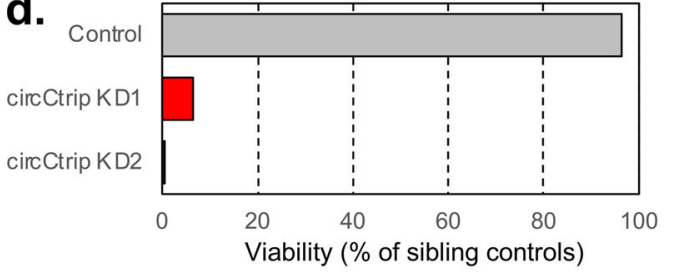

g.

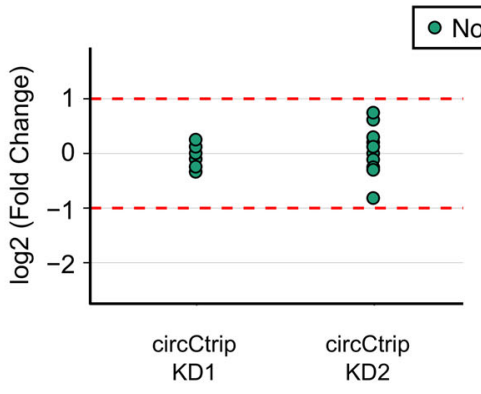

i.

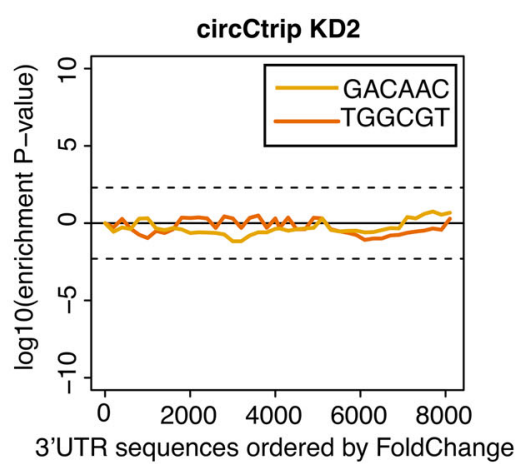

Fig. 4 (See legend on next page.) 
(see figure on previous page)

Fig. 4 circCtrip is essential for proper development. a Viability of circCtrip knockdown compared to their sibling controls. In green, percentage of pupae and in yellow 3rd instar larvae developed from sorted 1st instar larvae. circCtrip KD flies show more than 90\% of larval lethality when compared to the control flies (actin-Gal4). For each KD strain we normalized the data to the one of the siblings (GFP+) individuals $(n=3$ sets of 50 embryos each). $\mathbf{b}$ Expression of circCtrip at different developmental stages. We utilized the whole embryo, larva, or pupae as source of material and assessed the levels of the circRNAs by qRT-PCR using rp49 and tubulin as normalization control $(n=3$, error bars represent standard error of the mean). c Schematic representation of the additional shRNA (shRNA-2) designed against circCtrip. The new shRNA is shifted 3 bases in the $3^{\prime}$ direction of the target sequence. $\mathbf{d}$ Developmental viability (percentage of siblings control) of flies expressing the second shRNAs against circCtrip under the control of the actin-Gal4 promoter. e qRT-PCR evaluation of the efficiency of knockdown of the circRNA by the use of the different shRNA lines in the fly CNS. In all cases we utilized the elav-Gal4 driver to express two different shRNAs for circCtrip, RNA was prepared from heads. We then determined the efficiency of the knockdown by comparing to the levels of the assayed circRNA in the control strain (elav-Gal4). Data was normalized to $18 \mathrm{~S}$ rRNA and $r p 49$ ( $n=3$, error bars represents standard error of the mean). $\mathbf{f} 3^{\prime}$ RNA-seq data was used to determine the expression level of ctrip mRNA in circCtrip KD lines ( $n=3$, error bars represent standard error of the mean). $\mathbf{g}$ Expression of putative off-targets genes in both circCtrip KD lines. Potential off-targets genes were selected using blast of the shRNA sequence against the drosophila transcriptome. $3^{\prime}$ RNA-seq data was used to determine the expression level of each putative off-targets gene relative to control line (elav-Gal4, $n=3$ ). Differential gene expression analysis was performed using a generalized linear model with negative binomial distribution (see Materials and methods for details). None of the detected offtarget mRNAs displayed statistically significant differences (log2 (fold change) $>1$ and corrected $p$ value $<0.05$ ). $X$-axis presents fold change as log 2 of the fold change. $\mathbf{h}$, i Assessment of off-targets of the indicated shRNAs by SYLAMER. Seed enrichment was calculated for the genes differentially expressed upon downregulation of circCtrip using each shRNA. shRNA and shRNA* seed sequences shown in different colors respectively. Genes were sorted from downregulated to upregulated. For both knockdowns we did not observed any shRNA seed enriched among the downregulated genes.

type of manipulation might also alter the levels of the mRNA generated from the locus. Perturbation of the expression of the linear RNA might reflect a function in cis of the circRNA production (regulation of linear mRNA production) or an unwanted effect of the manipulation. This makes deletion experiments non-optimal, in particular for circRNAs with high production rates that can potentially have functions in cis and in trans.

shRNAs target circRNAs after production allowing the study of trans-like functions of these molecules. However, shRNA-mediated silencing has potential problems, chiefly the targeting of RNAs other than the desired circRNA. In particular, depending on their sequence, shRNAs can target linear RNAs generated from the circRNA-hosting locus. Limited complementarity (12-13 bases) can in some cases be enough to induce slicing of an mRNA or can inhibit protein production if the shRNA acts as a miRNA. Although this is a problem in mammals, it is less likely to be an issue in Drosophila in which the miRNA and shRNA pathways are compartmentalized as the AGO and DICER proteins are different in the two pathways ${ }^{42}$. Indeed, we did not observe miRNA-like downregulation of the hosting or other mRNAs when any of our shRNAs were expressed. In addition, we did not see any changes in the levels of mRNAs sharing more than 12 bases complementarity with the expressed shRNA. These results strongly suggest that the shRNAs used here have no offtarget effects, although it is not possible to completely rule out this possibility. When implementing our shRNAbased approach, it will be necessary to perform off-target analysis such as those described here.

Additional and concurrent knockdown or knockout approaches could corroborate the phenotypes we observed upon circRNA knockdown. These approaches could include the generation of additional shRNAexpressing strains that target slightly different regions of the back-splice junctions, the use of other systems for degrading specific RNAs (e.g., Cas $13 \mathrm{~b}^{43}$ ), or the generation of flies with the circRNA knocked down by a different method (e.g., by CRISPR deletion of inverted repeats of RNA binding protein sites). The method described here constitutes a straightforward method for knockdown of circRNAs in a specific tissue or the whole organism that can be used as a first step in analysis of circRNA function.

Importantly, the observed knockdown is strong (more than 5 -fold and in most cases almost total) and can be restricted in time and space. Spatial resolution is easy to achieve in Drosophila by the use of different GAL4 drivers $^{44}$. Indeed, here we restricted the expression of the shRNAs to neuronal tissue. Several systems that allow for temporal expression exist in Drosophila. The most commonly used involves the reversible inhibition of GAL4based transgene expression by a temperature sensitive (ts) GAL80 inhibitor ${ }^{44}$. We do not believe that this strategy of GAL4-induction (and hence silencing) will work to silence circRNAs, as circRNA expression dramatically increases at higher $\left(29^{\circ} \mathrm{C}\right)$ temperatures ${ }^{6}$. However, other systems such as the Geneswitch should efficiently induce the expression of UAS-based shRNA in a temporally regulated fashion ${ }^{44}$.

In sum, this manuscript describes a platform for silencing of circRNA expression that includes methods for determination of the accuracy and specificity of the shRNA reagents. We generated several Drosophila lines in which specific circRNAs were targeted for degradation using shRNAs. We observed that circCtrip is essential for proper fly development, whereas the other four circRNAs evaluated might regulate gene expression. In the future it 
will be interesting to test these flies for different phenotype consequences of the misregulated gene expression. Together, this works presents a new approach for assessing the functions of circRNAs in vivo.

\section{Materials and methods Fly strains}

Wild-type flies that we used in this study is $w^{1118}$ strain (Bloomington Drosophila Stock Center Indiana, USA). For constitutive knockdown of circRNAs we utilized the actin-Gal4 driver (stock number 3953, Bloomington Drosophila Stock Center, Indiana, USA). For neuralspecific knockdowns we utilized an elav-Gal4; UAS Dcr2 strain that was generated by using elav-Gal4 (stock number 458, Bloomington Drosophila Stock Center, Indiana, USA) and UAS-Dcr 2 flies. All crosses were performed and raised at $25^{\circ} \mathrm{C}$.

\section{Generation of shRNA lines}

To generate UAS-shcircRNA flies we utilized oligonucleotides with perfect 21-nucleotide complementary sequence to the circRNA junction, annealed them, and ligated into the linearized Valium20 vector with EcoR1 and Nhe1 restriction enzymes. Colonies were screened by PCR and the plasmid was purified and sequenced from positive colonies. These plasmids were sent for injection to BestGene Inc (CA, USA).

\section{Real-time PCR analysis}

Total RNA was extracted from adult fly heads using TRI Reagent (Sigma) and treated with DNase I (NEB) following the manufacturer's protocol. cDNA was synthesized from this RNA (using iScript and random primers, Bio-Rad) and was utilized as a template for quantitative real-time PCR performed with the C1000 Thermal Cycler Bio-Rad. The PCR mixture contained Taq polymerase (SYBR green Bio-Rad). Cycling parameters were $95^{\circ} \mathrm{C}$ for $3 \mathrm{~min}$, followed by 40 cycles of $95^{\circ} \mathrm{C}$ for $10 \mathrm{~s}, 55^{\circ} \mathrm{C}$ for $10 \mathrm{~s}$, and $72^{\circ} \mathrm{C}$ for $30 \mathrm{~s}$. Fluorescence intensities were plotted versus the number of cycles by using an algorithm provided by the manufacturer. Primer efficiency was determined for all primers described in this study and was taken into account for the relative expression calculation. The sequences of all the primers used in this assay are detailed in Supplementary Table S1.

\section{Assessment of developmental lethality}

Ten homozygous shcircRNA male flies were crossed with 10 virgin female actin-Gal4 flies and transferred to new bottles every 3 days. The F1 progeny was separated based on their genotype (indicated by the presence of the marker/balancer $\mathrm{CyO}$ ) and the number of males and females counted. We performed this assessment for each bottle for 9 days or until the totality of the F1 eclose.

\section{RNA libraries preparation for RNA-seq analysis}

Total RNA was extracted using Trizol reagent (Sigma) and treated with DNase I (NEB) following the manufacturer's protocol. Then, $0.5 \mu \mathrm{g}$ of total RNA was fragmented in FastAP buffer (Thermo Scientific) for $3 \mathrm{~min}$ at $94{ }^{\circ} \mathrm{C}$, then dephosphorylated with FastAP, cleaned (using SPRI beads, Agencourt) and ligated to a linker1 (5Phos/AXXXXX XXXAGATCGGAAGAGCGTCGTGTAG/3ddC/, where XXXXXXXX is an internal barcode specific for each sample), using T4 RNA ligase I (NEB). Ligated RNA was cleaned-up with Silane beads (Dynabeads MyOne, Life Technologies) and pooled into a single tube. This mix then polyA $^{+}$selected (using Oligo(dT) beads, Invitrogen), RT was then performed for the pooled sample, with a specific primer (5'-CCTACACGACGCTCTTCC-3') using AffinityScript Multiple Temperature cDNA Synthesis Kit (Agilent Technologies). Then, RNA-DNA hybrids were degraded by incubating the RT mixture with $10 \% 1 \mathrm{M} \mathrm{NaOH}$ (e.g. $2 \mathrm{uL}$ to $20 \mathrm{uL}$ of RT mixture) at $70^{\circ} \mathrm{C}$ for $12 \mathrm{~min}$. $\mathrm{pH}$ was then normalized by addition of corresponding amount of $0.5 \mathrm{M}$ $\mathrm{AcOH}$ (e.g. 4 ulL for $22 \mathrm{uL}$ of $\mathrm{NaOH}+\mathrm{RT}$ mixture). The reaction mixture was cleaned up using Silane beads and second ligation was performed, where $3^{\prime}$ end of cDNA was ligated to linker2 (5Phos/AGATCGGAAGAGCAC ACGTCTG/3ddC/) using T4 RNA ligase I. The sequences of linker1 and linker2 are partially complementary to the standard Illumina read1 and read2/barcode adapters, respectively. Reaction Mixture was cleaned up (Silane beads) and PCR enrichment was set up using enrichment primers 1 and 2:

(5'AATGATACGGCGACCACCGAGATCTACACTC TTTCCCTACACGACGCTCTTCCGATCT-3', 5'-CAA GCAGAAGACGGCATACGAGATXXXXXXXXGTGAC TGGAGTTCAGAC

GTGTGCTCTTCCGATCT- 3 ', where XXXXXXX is barcode sequence) and Phusion HF MasterMix (NEB). 12 cycles of enrichment were performed. Libraries were cleaned with $0.7 x$ volume of SPRI beads. Libraries were characterized by Tapestation. RNA was sequenced as paired-end samples, in a NextSeq 500 sequencer (Illumina).

\section{Western blot analysis}

Fly heads (20 heads per sample) were collected on dry ice. Heads were homogenized in RIPA lysis buffer $(50 \mathrm{mM}$ Tris- $\mathrm{HCl}$ at $\mathrm{pH}$ 7.4, $150 \mathrm{mM} \mathrm{NaCl}, 1 \mathrm{mM}$ EDTA, $1 \% \mathrm{NP}-$ 40 0.5\% Sodium deoxycholate, and $0.1 \%$ sodium dodecyl sulfate (SDS), $1 \mathrm{mM}$ DTT, supplemented by protease inhibitor cocktail and phosphatase inhibitors) using a motorized pestle. Head lysates were then centrifuged at maximum speed for $10 \mathrm{~min}$ and the supernatant was saved. Lysates were boiled with protein sample buffer (Bio-Rad) and resolved by Criterion XT Bis-Tris gels (BioRad). Antibodies used for western blotting: rabbit anti 
PKN was kindly provided by Prof. Rui Goncalo Martinho, (University of Algarve, Portugal, 1:1000), rabbit anti PlexA was kindly provided by Prof. Liqun Luo (Howard Hughes medical institute, Stanford university, 1:1000), mouse anti-tubulin (DM1A; SIGMA, 1:30,000).

\section{Determination of developmental stage at which circRNA KD provokes lethality}

We used actin-Gal4/CyO-GFP to achieve ubiquitous knockdown of circRNAs and score out non-Gal4 expressing siblings by excluding GFP-expressing larvae. Virgin females of Gal4 and males of UAS-shRNA were crossed a day before. On the day of experiment, we transferred the crossed flies to embryo collection chamber with yeast paste on sucrose agar plates. Three sets of embryos ( 100 each) were collected, aligned in a straight line against a coverslip on a sucrose agar plate. Thus, aligned embryos are allowed to develop and hatch into larvae. We then identified, counted and separated based on the presence of GFP the 1st instar larvae. Then, we counted the number of 3rd instar larvae and the number of larvae that pupated. The data corresponding to the Non-Cyo (shcircRNA KD) was then normalized to the one of their siblings, averaged and plotted.

\section{Gene expression analysis}

RNA-seq reads were aligned to the genome and transcriptome $(\mathrm{dm} 3)$ using tophat ${ }^{45}$. Gene expression levels from $3^{\prime}$ DGE experiments were determined using ESAT tool $^{37}$ and differential expression analysis was performed with DEseq2. We considered genes with fold change $>1.5$ and $p$ value $<0.05$ as significantly changing. Actin-Gal4 flies were used as a control for the lines expressing shRNA under actin promoter. In order to clean non-specific effects, we excluded from downstream analyses genes that change in all the circRNA KD strains. elav-Gal4; UASDcr2 flies were used as a control for the lines expressing shRNA under elav promoter. clusterProfiler package was used for enrichment analysis of gene ontology of the differentially expressed genes. GO terms with $p$-value $<$ 0.1 (after FDR correction) were considered significant.

\section{Determination of potential off-targets}

We determined of targets using two different approaches. First, we use SYLAMER algorithm ${ }^{38}$ to check for general off-target effect of the shRNA in the $3^{\prime}$ UTR regions of genes differentially regulated upon the circRNA KD. To that end we sorted the genes by fold change (from downregulated to upregulated) and searched for enrichment of the potential miRNA seed sequences recognized by the shRNAs within their $3^{\prime}$ UTR sequences. Secondly, in order to obtain a list of general potential shRNA offtarget genes we blasted all shRNA sequences against the latest version of the Drosophila transcriptome. To this aim we ran locally the BLAST algorithm for short sequences against the fly transcriptome for each of the shRNAs sequence. We then used the $3^{\prime}$ RNA-seq data to determine the expression level of each putative off-targets gene relative to control line. To this aim we used the results from the differential expression analysis (see details above) and considered genes to be significantly changing when $\log 2$ (fold change) $>1$ or $<-1$ and $p$ adjusted value $<0.05$. A small group of off-target genes were not found in the sequencing data (not expressed) or removed from this analysis because they were considered outliers or too lowly expressed by the requirements of the algorithm ( $p$ adjusted value $=\mathrm{NA}$ by DeSeq2, outlier counts are detected by Cook's distance automatically by DeSeq2).

\section{Acknowledgements}

We thank all the members of the Kadener lab for helpful discussions. This work was funded by National Institute of Health [grants R01GM122406 and R01AG057700] to S.K.

\section{Author contributions}

N.R. designed the shRNAs and performed most of the experiments; I.P. wrote the manuscript and performed the computational analysis; A.K. performed the crosses and the developmental experiments; R.A. generated the pipelines and conceptual framework for performing the off-target assessments; O.B.

performed the western blots and generated the RNAseq libraries; S.K. designed the experiments, supervised the project and wrote the manuscript.

\section{Conflict of interest}

The authors declare that they have no conflict of interest.

\section{Publisher's note}

Springer Nature remains neutral with regard to jurisdictional claims in published maps and institutional affiliations.

Supplementary Information accompanies the paper at (https://doi.org/ 10.1038/s41421-020-0182-y).

Received: 21 January 2020 Accepted: 18 May 2020

Published online: 11 August 2020

\section{References}

1. Jeck, W. R. \& Sharpless, N. E. Detecting and characterizing circular RNAs. Nat. Biotechnol. 32, 453-461 (2014).

2. Patop, I. L., Wust, S. \& Kadener, S. Past, present, and future of circRNAs. EMBO J. 38, e100836 (2019).

3. Li, X., Yang, L. \& Chen, L. L. The biogenesis, functions, and challenges of circular RNAs. Mol. Cell 71, 428-442 (2018).

4. Kristensen, L. S. et al. The biogenesis, biology and characterization of circular RNAs. Nat. Rev. Genet. 20, 675-691 (2019).

5. Salzman, J., Chen, R. E., Olsen, M. N., Wang, P. L. \& Brown, P. O. Cell-type specific features of circular RNA expression. PLoS Genet. 9, e1003777 (2013).

6. Ashwal-Fluss, R. et al. circRNA biogenesis competes with pre-mRNA splicing. Mol. Cell 56, 55-66 (2014).

7. Kramer, M. C. et al. Combinatorial control of Drosophila circular RNA expression by intronic repeats, hnRNPs, and SR proteins. Genes Dev. 29, 2168-2182 (2015).

8. Liang, D. \& Wilusz, J. E. Short intronic repeat sequences facilitate circular RNA production. Genes Dev. 28, 2233-2247 (2014)

9. Ivanov, A. et al. Analysis of intron sequences reveals hallmarks of circular RNA biogenesis in animals. Cell Rep. 10, 170-177 (2015). 
10. Zhang, X. O. et al. Complementary sequence-mediated exon circularization. Cell 159, 134-147 (2014).

11. Starke, S. et al. Exon circularization requires canonical splice signals. Cell Rep. 10, 103-111 (2015)

12. Conn, S. J. et al. The RNA binding protein quaking regulates formation of circRNAs. Cell 160, 1125-1134 (2015).

13. Pamudurti, N. R. et al. Translation of circRNAs. Mol. Cell 66, 9-21 e27 (2017).

14. Legnini, I. et al. Circ-ZNF609 is a circular RNA that can be translated and functions in myogenesis. Mol. Cell 66, 22-37 (2017).

15. Yang, $Y$. et al. Extensive translation of circular RNAs driven by N6methyladenosine. Cell Res. 27, 626-641 (2017).

16. Rybak-Wolf, A. et al. Circular RNAs in the mammalian brain are highly abundant, conserved, and dynamically expressed. Mol. Cell 58, 870-885 (2015).

17. Veno, M. T. et al. Spatio-temporal regulation of circular RNA expression during porcine embryonic brain development. Genome Biol. 16, 245 (2015).

18. Westholm, J. O. et al. Genome-wide analysis of drosophila circular RNAs reveals their structural and sequence properties and age-dependent neural accumulation. Cell Rep. 9, 1966-1980 (2014).

19. You, X. et al. Neural circular RNAs are derived from synaptic genes and regulated by development and plasticity. Nat. Neurosci. 18, 603-610 (2015).

20. Cortes-Lopez, M. et al. Global accumulation of circRNAs during aging in Caenorhabditis elegans. BMC Genom. 19, 8 (2018).

21. Gruner, H., Cortes-Lopez, M., Cooper, D. A., Bauer, M. \& Miura, P. CircRNA accumulation in the aging mouse brain. Sci. Rep. 6, 38907 (2016).

22. Piwecka, M. et al. Loss of a mammalian circular RNA locus causes miRNA deregulation and affects brain function. Science 357, eaam8526 (2017).

23. Hansen, T. B. et al. Natural RNA circles function as efficient microRNA sponges. Nature 495, 384-388 (2013).

24. Memczak, S. et al. Circular RNAs are a large class of animal RNAs with regulatory potency. Nature 495, 333-338 (2013).

25. Kleaveland, B., Shi, C. Y., Stefano, J. \& Bartel, D. P. A network of noncoding regulatory RNAs acts in the mammalian brain. Cell 174, 350-362 (2018).

26. Du, W. W. et al. Foxo3 circular RNA retards cell cycle progression via forming ternary complexes with p21 and CDK2. Nucleic Acids Res. 44, 2846-2858 (2016).

27. Patop, I. L. \& Kadener, S. circRNAs in cancer. Curr. Opin. Genet. Dev. 48, 121-127 (2018).

28. Chen, Y. G. et al. Sensing self and foreign circular RNAs by intron identity. Mol. Cell 67, 228-238 (2017).
29. Li, X. et al. Coordinated circRNA biogenesis and function with NF90/NF1 10 in viral infection. Mol. Cell 67, 214-227 (2017).

30. Chen, Y. G. et al. N6-methyladenosine modification controls circular RNA immunity. Mol. Cell 76, 96-109 (2019).

31. Liu, C. X. et al. Structure and degradation of circular RNAs regulate PKR activation in innate immunity. Cell 177, 865-880 (2019).

32. Zimmerman, A. J. et al. A psychiatric disease-related circular RNA controls synaptic gene expression and cognition. Mol. Psychiatry https://doi.org/ 10.1038/s41380-020-0653-4 (2020).

33. Zheng, Q. et al. Circular RNA profiling reveals an abundant circHIPK3 that regulates cell growth by sponging multiple miRNAs. Nat. Commun. 7, 11215 (2016).

34. Zhang, Y. et al. The biogenesis of nascent circular RNAs. Cell Rep. 15, 611-624 (2016).

35. Xia, P. et al. A circular RNA protects dormant hematopoietic stem cells from DNA sensor cGAS-mediated exhaustion. Immunity 48, 688-701 (2018).

36. $\mathrm{Ni}, J$. Q. et al. A genome-scale shRNA resource for transgenic RNAi in Drosophila. Nat. Methods 8, 405-407 (2011).

37. Derr, A. et al. End Sequence Analysis Toolkit (ESAT) expands the extractable information from single-cell RNA-seq data. Genome Res. 26, 1397-1410 (2016).

38. van Dongen, S., Abreu-Goodger, C. \& Enright, A. J. Detecting microRNA binding and siRNA off-target effects from expression data. Nat. Methods $\mathbf{5}$, 1023-1025 (2008)

39. Xu, X. Z. et al. Retinal targets for calmodulin include proteins implicated in synaptic transmission. J. Biol. Chem. 273, 31297-31307 (1998).

40. Hueston, C. E. et al. Chromatin modulatory proteins and olfactory receptor signaling in the refinement and maintenance of fruitless expression in olfactory receptor neurons. PLoS Biol. 14, e1002443 (2016).

41. Yu, L., Zhou, Y., Cheng, S. \& Rao, Y. Plexin a-semaphorin-1a reverse signaling regulates photoreceptor axon guidance in Drosophila. J. Neurosci. 30, 12151-12156 (2010).

42. Forstemann, K., Horwich, M. D., Wee, L., Tomari, Y. \& Zamore, P. D. Drosophila microRNAs are sorted into functionally distinct argonaute complexes after production by dicer-1. Cell 130, 287-297 (2007).

43. Pickar-Oliver, A. \& Gersbach, C. A. The next generation of CRISPR-Cas technologies and applications. Nat. Rev. Mol. Cell Biol. 20, 490-507 (2019).

44. Duffy, J. B. GAL4 system in Drosophila: a fly geneticist's Swiss army knife. Genesis 34, 1-15 (2002).

45. Trapnell, C., Pachter, L. \& Salzberg, S. L. TopHat: discovering splice junctions with RNA-Seq. Bioinformatics 25, 1105-1111 (2009). 